\title{
Intrinsic, Light-Independent and Visual Activity-Dependent Mechanisms Cooperate in the Shaping of the Field Response in Rat Visual Cortex
}

\author{
Marian Tsanov and Denise Manahan-Vaughan \\ Learning and Memory Research, Medical Faculty, and International Graduate School for Neuroscience, Ruhr University, 44780 Bochum, Germany
}

\begin{abstract}
Do light intensity and behavioral state regulate synaptic plasticity in the visual cortex? We have shown previously that synaptic transmission in the visual cortex oscillates between elevated and depressed levels in accordance with the diurnal light- dark cycle. In this study, we examined the role of intrinsic, light-independent, and visual activity-driven sensory information on the field response during diurnal fluctuations, and examined the plasticity properties of the visual cortex under both conditions. Recordings were obtained from layer $2 / 3$ of the primary visual cortex, of adult freely moving Long Evans rats, after stimulation of the dorsal lateral geniculate nucleus. We observed that visual experience during different states of vigilance leads to increased responsiveness, and plastic changes, in the strength of connections among neurons, consistent with a naturalistic shift in the induction thresholds for synaptic plasticity. We identified this phenomenon as BDNF-dependent. We also found that gamma oscillatory activity, which increases during active visual exploration, is tightly associated with suppression of cortical field potentials, suggesting that coincident changes in synaptic responsiveness and gamma oscillatory levels may reflect mechanisms for optimal stimulus-feature encoding. Translating into an increased signal-to-noise ratio, field depression could thus alter the efficacy of cortical visual processing. These data indicate that the adult visual cortex serves as a synaptic network, where the ability to process visual stimuli is dynamically modified by active visual exploration and arousal states.
\end{abstract}

Key words: lateral geniculate nucleus; layer 2/3; thalamocortical synapse; synaptic plasticity; in vivo; visual cortex

\section{Introduction}

An important strategy in understanding how experience modifies cortical processing comprises investigations of the properties and functional consequences of synaptic plasticity in the behaving animal. Although substantial progress has been made in understanding how long-term potentiation and long-term depression may work together to enable information storage (Bear, 1996; Martin et al., 2000; Kemp and Manahan-Vaughan, 2004, 2005, 2007), very little is known about the physiological conditions that drive synaptic plasticity in the neocortex or visual cortex. Synaptic experience results in an alteration of neuronal properties, which may range from transient changes to permanent strengthening, or weakening, of synaptic connections. Neuronal responses vary as a function of network activities: a possibility supported by the observation that, in the primary visual cortex, basal synaptic transmission oscillates between elevated and depressed states, which are driven, in turn, by activity phases (Tsanov and Manahan-Vaughan, 2007).

Light exposure also triggers the rodent endogenous circadian

Received Dec. 12, 2006; revised May 15, 2007; accepted May 16, 2007.

This work was supported by German Research Foundation (Deutsche Forschungsgemeinschaft) Grant SFB509/TP C13 (D.M.-V.). We are very grateful to Jens Klausnitzer and Dr. Arthur Bikbaev for technical assistance and advice. Correspondence should be addressed to Dr. Denise Manahan-Vaughan, Learning and Memory Research, Medical Faculty, Ruhr University, Forum Nord 0st 1/116, Universitaetsstrasse 150, 44780 Bochum, Germany. E-mail: dmv-igsn@rub.de.

DOI:10.1523/JNEUROSCI.1180-07.2007

Copyright $\odot 2007$ Society for Neuroscience $\quad 0270-6474 / 07 / 278422-08 \$ 15.00 / 0$ clock (suprachiasmatic nuclei of the hypothalamus), which in turn dynamically modulates many behavioral and physiological functions in mammals (Rusak and Zucker, 1979; Meijer and Rietveld, 1989; Ralph et al., 1990). Cortical neuronal responsiveness is altered by behavioral states (Steriade et al., 1998). It displays the highest amplitudes during slow-wave sleep and is disrupted after natural arousal, or during forebrain activation elicited by stimulation of the brainstem reticular formation (Castro-Alamancos and Connors, 1996; Timofeev and Steriade, 1998; Steriade, 2000, 2001). To address the manner in which visual experience increases field responses in the visual cortex, we examined the factors that are related to diurnal oscillations of basal synaptic transmission in the visual cortex of freely moving adult animals (Tsanov and Manahan-Vaughan, 2007). We also investigated the role of brain derived neurotrophic factor, because of its known role in visual cortex plasticity phenomena (Huber et al., 1998). Our results demonstrate that diurnal variations in illuminance that trigger prolonged changes in synaptic strength are coupled to activity phases, network activity, and the degree of active visual processing in the visual cortex. These factors strongly influence the ability of the animal to generate synaptic plasticity during active vigilant states.

Active visual processing in the visual cortex may be mediated by high-frequency oscillations, which comprise an important network property for mediating the perceptual functions of the neocortex (Singer and Gray, 1995). On a population level, rapid and reliable transmission is enabled by the activity of gamma 
(30-80 Hz) oscillations (Lumer et al., 1997; Fries et al., 2001). On a synaptic level, the physiological response of the visual cortex to sensory encoding, evoked by high-contrast features, is mediated through synaptic depression (Varela et al., 1997), and leads to a dramatic increase in the sensitivity of a neuron to subtle changes in the firing pattern of its afferents (Abbott et al., 1997). This suggests that a correlation exists between synaptic depression and changes in fast oscillatory activity in the visual cortex: a possibility which we also addressed in this study.

\section{Materials and Methods}

Surgical preparation. Under sodium pentobarbitone anesthesia (Nembutal; 40 mg/kg, i.p.), 7- to 9-week-old male Long Evans rats (Charles River Laboratories, Sulzfeld, Germany) underwent implantation of a monopolar recording and a bipolar stimulating electrode (made from $0.1 \mathrm{~mm}$ diameter Teflon-coated stainless-steel wire).

A small hole was drilled ( $1 \mathrm{~mm}$ diameter) for a recording electrode 7.1-7.3 mm posterior to bregma and 3.4-3.6 $\mathrm{mm}$ lateral to the midline, corresponding to primary visual cortex in the rat (Zilles, 1985). A second drill hole (3.8 $\mathrm{mm}$ posterior to bregma; $3.3-3.4 \mathrm{~mm}$ lateral to midline) was created for the placement of a bipolar stimulating electrode. The dura was pierced through both holes using a needle. The recording electrode was lowered $200-250 \mu \mathrm{m}$ from the dural surface to reach the supragranular layer $2 / 3$. The stimulating electrode was positioned in the dorsal lateral geniculate nucleus (dLGN), ipsilateral to the hemisphere in which visual cortex recordings were obtained. The depth comprised 3.7$4.7 \mathrm{~mm}$ from the dural surface, corresponding to the position of the anterior pole where optic radiation fibers are bundled in close proximity (Hughes, 1977; Molnar et al., 1998). Final positions of the stimulating and recording electrodes were then determined by maximizing the amplitude of the field potential (FP) recorded in the visual cortex in response to electrical stimulation of the dLGN. Monopolar recordings from the primary visual cortex were made relative to ground and reference screws inserted into the contralateral parietal and frontal bone plates. Once verification of the location of the electrodes was complete, the entire assembly was sealed and fixed to the skull with dental acrylic (Paladur; Heraeus Kulzer, Wehrheim, Germany). The animals were allowed $10 \mathrm{~d}$ to recover from surgery before experiments were conducted. Throughout the experiments, the animals moved freely within the recording chamber $(40 \times 40 \times 40 \mathrm{~cm})$, because the implanted electrodes were connected by a flexible cable and swivel connector to the stimulation unit and amplifier. Aside from the insertion of the connector cable at the start of the experiment, disturbance of the animals was kept to an absolute minimum. Throughout the experiments, the electroencephalogram (EEG) of each animal was continuously monitored.

Measurement of evoked potentials. The field EPSP (fEPSP) slope was used as a measure of excitatory synaptic transmission in the V1 region. To obtain these measurements, an evoked response was generated in the dLGN by stimulating at low frequency $(0.025 \mathrm{~Hz})$ with single biphasic square-wave pulses of $0.1 \mathrm{~ms}$ duration per half wave, generated by a constant current isolation unit. For each time-point measured during the experiments, five records of evoked responses were averaged. The fEPSP was measured as the maximal slope through the five steepest points obtained on the negative deflection of the potential. By means of inputoutput curve determination, the maximum fEPSP was found, and during experiments all potentials used as baseline criteria were evoked at a stimulus intensity that produced $40 \%$ of this maximum $(100-400 \mu \mathrm{A})$.

The animals were kept in a 12/12 h light-dark cycle of low- (0.1-10 lux) and high-illuminance (350-400 lux) conditions. Circadian time was based on Zeitgeber time (ZT), with ZT 0 denoting lights on and ZT 12 denoting lights off. Basal synaptic transmission (baseline) measurements were made under low illuminance conditions during the end of the dark period of the cycle. Exactly $24 \mathrm{~h}$ later, measurements were recorded at the same circadian time and conditions. Baseline responses were collected for 30-60 min before changing of illuminance conditions. For the experiments with prolonged dark exposure, the light was kept off at ZT 0 , on the day of experiment, and the animals underwent low-illuminance exposure until the end of the recording.
Recording environment and novel objects. The recording chamber consisted of a gray Perspex box $(40 \times 40 \times 40 \mathrm{~cm})$ with a translucent red perspex door. Novel objects consisted of large objects $(10 \times 10 \times 3 \mathrm{~cm})$ with different colors (black, white, green) and shapes (cylindric or square). In some cases (for the prolonged dark-exposure experiments) the objects were fluorescent. Illuminance of objects and environments was assessed using a luxmeter (MS-1300) with a sensitivity range of $0-50000$ lux. The luxmeter was placed on the floor of the recording chamber and pointed at the light source. The sensor unit possessed a photosensitive surface, which recorded the total amount of falling light. A possible change in illuminance derived from the placement of a fluorescent object in the recording box was assessed by placing the meter at a distance of 15-20 cm from, although pointing directly at the object.

Analysis of electrophysiological data. The baseline fEPSP data were obtained by averaging the response to stimulation of the dLGN to obtain five sweeps at $40 \mathrm{~s}$ intervals, every $5 \mathrm{~min}$ over a period of $30 \mathrm{~min}$. Electrophysiological data were then expressed as mean percentage of baseline fEPSP reading \pm SEM. Statistical significance was estimated by using factorial ANOVA and post hoc Student's $t$ and Bonferroni tests. Using factorial ANOVA, we estimated the effects of the main factors, illuminance (two levels, high and low illuminance) and drug application (two levels, yes and no) on the field potential amplitude. Additionally, we estimated the effect of time on the field potential values compared with the baseline period (composed of the first six time points). The probability level interpreted as statistically significant was ${ }^{\star} p<0.05$.

Analysis of network activity. The intracortical EEG was obtained via recordings from the supragranular layer $2 / 3$ of V1. EEG was sampled at $0.5 \mathrm{kHz}$ and stored on a hard disc for additional off-line analysis. To evaluate delta $(1-3.5 \mathrm{~Hz})$, theta $(4-10 \mathrm{~Hz})$, alpha $(10-13 \mathrm{~Hz})$, betal $(13.5-18 \mathrm{~Hz})$, beta2 $(18.5-30 \mathrm{~Hz})$, and gamma $(30-100 \mathrm{~Hz})$ oscillatory activity during the course of the experiment, $4 \mathrm{~s}$ long epochs, $1 \mathrm{~s}$ after each test pulse, were selected. Fourier analysis of artifact-free epochs was performed with a Hanning window function using Spike2 software (Cambridge Electronic Design, Cambridge, UK). The absolute values of spectral power for each individual animal were transformed into relative ones (with the mean value for the baseline preinjection period taken as 100\%) that were then used further for statistics. For each time point, the results of Fourier analysis of five epochs were averaged. The statistical treatment and analysis of data included the calculation of descriptive statistics (mean, SEM) and ANOVA. ANOVA was used to estimate the effects of illuminance changes (two levels, high and low illuminance) and of exploratory activity (two levels, active and inactive behavioral state). For the correlation analyses we used Pearson's coefficient of comparison.

Postmortem verification of electrode site. At the end of the study, brains were removed for histological verification of electrode localization. Brain sections $(16 \mu \mathrm{m})$ were embedded in paraffin, stained according to the Nissl method using 1\% toluidine blue, and then examined using a light microscope. Brains in which an incorrect electrode localization was found were discarded from the study.

Compounds and drug treatment. K252a (Biomol, Plymouth Meeting, PA), a broad-spectrum inhibitor for Trk receptor tyrosine kinases, was prepared at a concentration of $40 \mu \mathrm{M}$ with dimethylsulfoxide (DMSO). The final concentration of DMSO was $0.4 \%(\mathrm{v} / \mathrm{v})$. Anti-tyrosine kinase B (TrkB) IgG1 (clone 47; BD Transduction Laboratories, San Jose, CA) was prepared at a concentration of $1 \mu \mathrm{g} / \mu \mathrm{l}$, dissolved in Ringer's solution, and stored at $4^{\circ} \mathrm{C}$ until use. K252a, anti-TrkB IgG1, or vehicle were injected in a $5 \mu \mathrm{l}$ volume over a $5 \mathrm{~min}$ period via a Hamilton syringe. The Hamilton syringe was connected by means of a flexible polyurethane tube to an injection cannula that was inserted into the permanently implanted cannula. Antagonist or vehicle injection was performed $20 \mathrm{~min}$ before the illuminance shift (ZT 0) to enable diffusion from the lateral cerebral ventricle into CSF circulation to occur. Throughout the light phase, injections were administered twice at $20 \mathrm{~min}$ intervals. After the change in illuminance, measurements were then taken at $T=5,10,15$ and then $15 \mathrm{~min}$ intervals up to $4 \mathrm{~h}$, with additional measurements taken 24 through 25 h later.

Estimation of behavioral state. The exact estimation of the behavioral state, according to which the animals were divided into two groups, was based on off-line analysis. The animals grouped as exhibiting an inactive 
behavioral state were characterized by locomotor activity at $<20 \mathrm{~cm} / \mathrm{min}$ and theta and delta spectral power throughout the recordings at $<150 \%$, compared with baseline measurements. The rats grouped as having an active behavioral state were characterized by locomotor activity at $>30 \mathrm{~cm} / \mathrm{min}$ and theta and delta spectral power throughout the recordings at $>250 \%$, compared with baseline measurements.

\section{Results}

Intrinsic, light-independent and visual activity-dependent mechanisms cooperate in the shaping of the diurnal field potential augmentation

The field response in the visual cortex undergoes diurnal augmentation after exposure to light at the end of the dark phase of the light-dark cycle (Tsanov and Manahan-Vaughan, 2007). The first goal of our study was to clarify whether this phenomenon may represent an intrinsic circadian thalamocortical change or whether it could be the result of visual activity. To address this question, two groups of rats were introduced for the first time to the recording chamber (naive rats) under low illuminance conditions (0.1-10 lux). After a $30 \mathrm{~min}$ test-pulse period, illuminance was switched to daylight conditions (300400 lux) in the first group, whereas the second group remained under low-illuminance conditions until the end of the experiment (Fig. 1). Thus, the visual cortex of the different rat groups was involved to a different extent in the perception of the new environment.

We found that a significant FP augmentation occurred, which commenced at a similar point in the $24 \mathrm{~h}$ cycle in both groups (Fig. 1A) (ANOVA; $F=5.78, p<$ $0.001, n=6$ for the light-exposed group; $F=1.9, p<0.05$ for the group with prolonged dark exposure). The extent of FP augmentation was significantly less in the animals that were continuously exposed to low-illuminance conditions, compared with the highilluminance-exposed group (Fig. 1A) (ANOVA, $F=2.1, p<$ $0.05 ; n=4$ for the first group and $n=6$ for the second group).

This result reveals an intrinsic, light-independent, circadian oscillation of neocortical field responses and suggests the role of a visually driven component in the composition of the final FP amplitude.

To investigate the possibility that high-frequency oscillations that are induced by visual activity (Singer and Gray, 1995) may influence synaptic plasticity in the visual cortex, we compared the expression of gamma oscillations in both groups. Daylight conditions elicited a prominent increase of gamma activity (30-100 $\mathrm{Hz}$ ) in the first group (Fig. $1 B, C$ ), in correlation with the increased locomotor activity (Fig. $1 D$ ). Later, during the inactive state of the animal, gamma activity decreased significantly below the control period levels (ANOVA, $F=3.72, p<0.001 ; n=6$ ). For the dark-exposed group, no elevation of gamma activity was observed (Fig. 1C). The factor "light," calculated with a post hoc Bonferroni test for each time point, revealed a significance of $p<$ Error bars indicate SEM.

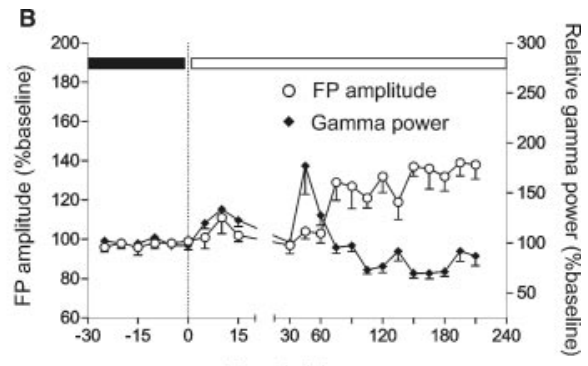

D

Time (min)
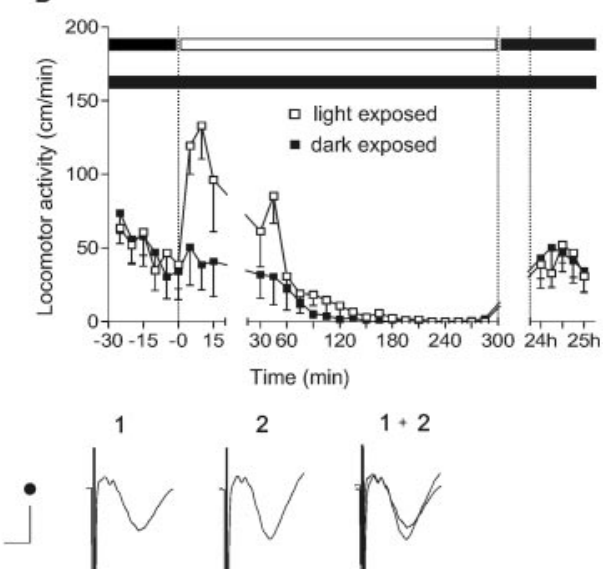

Figure 1. The experience-dependent increase of cortical synaptic transmission is mediated by cortical gamma activity in vency os llations during visual exploration under high-illuminance conditions. C, Absolute gamma power in the same groups of animals. In contrast to the light-exposed animals, the roup of animals kept in prolonged dark exposure revealed no significant

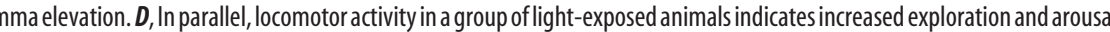

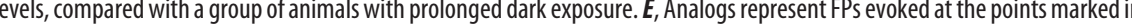
the figures. Calibration: $5 \mathrm{~ms}, 1 \mathrm{mV}$. Left group of analogs represents an example of the FP change in light-exposed animals; the right group shows an example of the FP change in animals that underwent prolonged dark exposure. ${ }^{*} p<0.05$; $^{* * *} p<0.001$.

0.05 for the value measured at 10th minute, and $<0.001$ for the values at fourth and sixtieth minutes after the start of the light exposure. The increase in gamma power was consistent across all animal groups, although occurring individually at different time points. Interestingly, locomotor activity (Tsanov and ManahanVaughan, 2007) reflected changes of exploratory behavior that were similar to those seen in gamma oscillations. Augmentation above the baseline period was not observed for the group of animals with prolonged dark exposure (Fig. 1D).

The transition into the inactive state was marked by an augmentation of low-frequency spectral power. A maximal increase in absolute power of delta $(1-3.5 \mathrm{~Hz})$ and theta $(4-10 \mathrm{~Hz})$ activity in the beginning of the nonaroused phase was equivalent in both groups (for theta, $p>0.1,3.182 ; n=4$ for the first group and $n=6$ for the second group; for delta, $p>0.1, F=2.452 ; n=$ 4 and $n=6$ ) (data not shown).

Subsequently, during the inactive phase of the animals, cortical synaptic transmission was significantly augmented in both groups, but the synaptic modification elicited in dark-exposed animals was proportionally less (Fig. $1 B$ ). Together, these data suggest that the diurnal elevation in visual cortex field potential is composed of two components: a light-independent intrinsic 

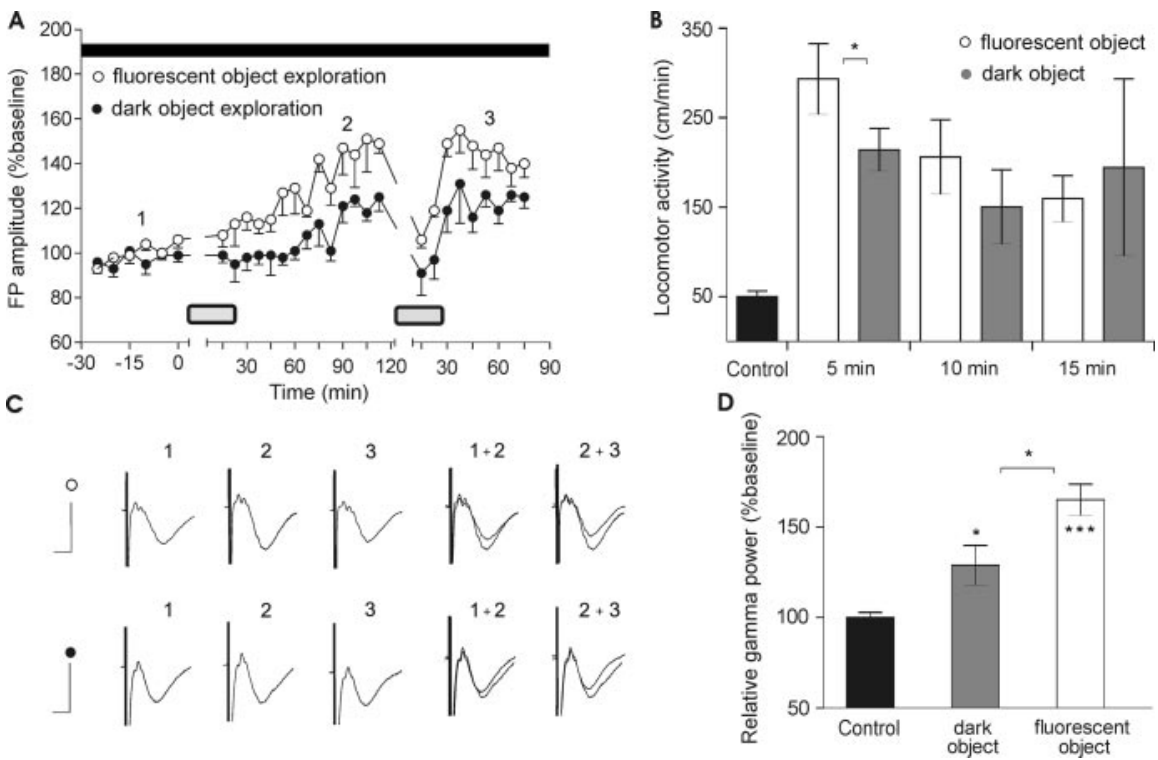

Figure 2. The elevation of basal synaptic transmission does not reflect a passive response to high illuminance conditions. $\boldsymbol{A}$, Maximal FP augmentation (140-150\%) occurs in the absence of light exposure. Animals were introduced to a toy (during the periods marked by gray rectangles on the graph). The first exposure began at time 0 for $15 \mathrm{~min}$. $\boldsymbol{B}, \mathrm{A}$ low-contrast object elicited a similar amount of exploration, measured by the locomotor activity (centimeters per minute) and compared with the baseline period (black bar) under dark conditions. Analysis from the first 5 min immediately after the introduction of the object showed a slightly increased activity for the high-contrast object (white bars) compared with the low-contrast one (gray bars). Nonetheless, overall locomotor activity for all three periods for both objects do not differ significantly. C, Analogs represent FPs evoked at the points marked in the figures. Calibration: $5 \mathrm{~ms}, 1 \mathrm{mV}$. D, Relative gamma power in the same groups of rats reflects the level of visual exploration induced by the objects presentation. The low-contrast object (gray bar) drives oscillatory activity to a significantly higher extent than the control level (black bar), and to a significantly lower extent than the high contrast-induced gamma augmentation (white bar). ${ }^{*} p<0.05 ;{ }^{* *} p<0.001$. Error bars indicate SEM.

mechanism on which visual activity-dependent modulation is superimposed.

\section{Active visual processing in the visual cortex is required for illuminance-induced changes in synaptic strength}

The plasticity we observed in the visual cortex may correspond to a passive response generated simply by illuminance levels, or may correlate with active visual processing. To address this issue, novel objects were presented to the animals during their active state (Fig. 2A). Under darkness conditions (0.1-10 lux), we introduced a high-contrast object (fluorescent toy). The illuminance levels in the recording chamber were not altered as a result. At the same time, we introduced a second group of rats to a novel low-contrast object (nonfluorescent toy) under the same illuminance conditions and during the same circadian period. The two types of toys used in our experiments were with the same shape, size and texture, except that one was fluorescent and high contrast, whereas the other was nonfluorescent and low contrast.

During the aroused phase, a significant increase in FP amplitude was recorded in the visual cortex of the group presented with the high-contrast object compared with the second group (lowcontrast object), where the ability of the visual system to detect stimulus features was decreased (Fig. 2A) (ANOVA, $F=2.37$, $p<0.05 ; n=4)$. Furthermore, during the nonaroused phase of the animals, when cortical synaptic transmission was augmented, the synaptic modification elicited in the group with the lowcontrast object was proportionally less. On a second occasion, a fluorescent toy was presented for $15 \mathrm{~min}$ after the animals entered the nonaroused state. When the object with the same contrast features (as that used at the beginning of the recording session) was presented again during the inactive state, short-term depres- sion occurred. After presentation, visual cortex synaptic transmission gradually increased compared with the synaptic transmission of animals that were introduced to a nonfluorescent object. The enhancement was proportional during active and subsequent inactive states, when FP amplitude was elevated.

Our data suggest that plastic changes do not arise as a passive response of the visual cortex to the light conditions, but crucially depend on visual activity of the animal and proper encoding of visual stimuli. In agreement with this idea is the observation that the levels of exploratory activity do not differ between the rats shown the high-contrast versus lowcontrast object $(n=5)$ (Fig. $2 B)$ and at the same time gamma oscillations are significantly higher for the group exposed to the fluorescent toy (Fig. 2D) ( $t$ test, $p>0.05$; $n=5)$. The locomotor activity was monitored every 5 min during of the object presentation (15 min total). Detailed analysis of the locomotor activity shows higher values for the fluorescent object immediately after its introduction (in the first $5 \mathrm{~min}$ ), corresponding to the faster effect of visual cues on the animal's arousal (Fig. $2 B)(t$ test, $p<0.05 ; n=5)$. Overall the locomotion-measured arousal for all 15 min does not differ significantly (Fig. $2 B$ ) ( $t$ test, $p>0.05 ; n=5$ ), suggesting that nonvisually guided exploratory activity is insufficient to produce the same amount of augmentation in visual cortex field response.

\section{TrkB activation is required for initiation of the diurnal field potential augmentation}

A challenging issue was to explore the mechanism responsible for diurnal augmentation of the visual cortex FP, induced by exposure to light at the end of the dark phase of the light-dark cycle. Two main aspects were considered in our investigation: (1) the factor that could drive the slow-onset potentiation seen, and (2) whether inhibiting this factor would affect the ability of the visual cortex to undergo diurnal oscillation.

Studies revealing BDNF-diurnal variations on the one hand, and the importance of neurotrophins in modeling the synaptic plasticity on the other, led us to suspect a role of BDNF in the observed diurnal shift in synaptic strength. When microinfused into the adult hippocampus in vivo, BDNF alone induces a slowonset persistent strengthening of synaptic transmission at perforant path-granule cell synapses (Messaoudi et al., 1998; Ying et al., 2002). This effect was termed BDNF-induced long-term potentiation (BDNF-LTP). Interestingly, the slow onset of BDNFLTP accurately fits to the profile of sinusoidal elevation that we observed in thalamocortical responses. In parallel, BDNF has been shown to facilitate LTP in the visual cortex (Akaneya et al., 1997; Huber et al., 1998). Pairing of BDNF with neuronal activity appears to be highly effective in inducing long-term synaptic potentiation (Figurov et al., 1996; Kovalchuk et al., 2002).

In the rat brain, in the absence of any experimental manipulation, the expression of BDNF and TrkB mRNA (Bova et al., 1998) undergoes diurnal oscillation. Furthermore, in the visual 
cortex, the tissue concentration of BDNF protein is greater in the middle of the light phase of the circadian cycle than in the middle of the dark phase (Pollock et al., 2001). Thus, we speculated that BDNF may comprise the factor that drives the FP augmentation that we had observed.

We tested this hypothesis and found that a block of TrkB receptors using the Trk receptor tyrosine kinase inhibitor, K252a, delayed the onset of FP augmentation (Fig. 3A) (ANOVA, $F=4.89, p<$ $0.001 ; n=4)$, suggesting a role for TrkB in this phenomenon. To verify this possibility, in another group of animals, we applied a specific antibody that blocks the function of TrkB receptors. Under these conditions, we found that FP augmentation was impaired, with recovery first becoming evident $\sim 4 \mathrm{~h}$ after the light exposure (Fig. $3 B$ ) (ANOVA, $F=3.76, p<$ $0.01 ; n=5)$.

We also explored slow and fast oscillatory activities after anti-TrkB IgG1 administration. Because the animals were familiar with the recording boxes, changing to daylight conditions (300-500 lux) did not result in highly increased exploratory and gamma oscillatory activity. Although under these conditions TrkB antagonism does not appear to mediate a significant change in gamma power, a brief discoordination between the time of light exposure and the fluctuation of the gamma oscillations was observed (Fig. $3 C, n=5$ ). BDNF signaling takes several minutes to affect the neuronal response in vivo (Messaoudi et al., 1998; Ying et al., 2002). Therefore, rapidly occurring network changes (oscillatory or stimulus evoked EPSPs) should be predominantly linked to glutamate- and GABAergic-mediated synaptic transmission.

\section{Visually evoked field depression correlates with gamma oscillatory activity}

Studies exploring dynamic gain control mechanisms in the visual cortex report that a cortical neuron with depressed synapses can detect synchronous presynaptic rate changes (Abbott et al., 1997). Therefore, the visually induced depression seen in our study (Fig. 2A) may be responsible for an enhancement of signalto-noise ratio, which in turn should lead to a higher degree of effectiveness in encoding stimulus features. This suggests that field depression is a general consequence of the activation of the arousal systems: in our experiments it can be seen in the transition from inactive to active, exploratory behavioral state. To address the question of whether this arousal-induced depression is influenced by illuminance conditions or object features, we introduced novel objects with different colors (black, white, green) or shapes (cylindric or square) to nonaroused animals under daylight conditions (Fig. 4A). On a second occasion, $2 \mathrm{~h}$ later, a presentation of a toy of different color and shape was conducted. The animals were divided into two groups depending on their locomotor activation: the aroused group (locomotor activity $>20 \mathrm{~cm} / \mathrm{min}$ ) and nonaroused group (locomotor activity $<20$ $\mathrm{cm} / \mathrm{min}$ ). The aroused group responded to a novel object exposure with a short-term depression of the field potential, which

Figure 3. Field potential augmentation is mediated through TrkB activation. $\boldsymbol{A}$, The TrkB antagonist K252a (40 $\mu \mathrm{m}$ ) blocks the

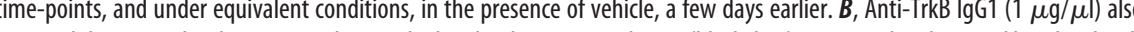
decreased gamma oscillatory activity. ${ }^{*} p<0.05$. D, Analogs represent FPs evoked at the points marked in the figures. Calibration:
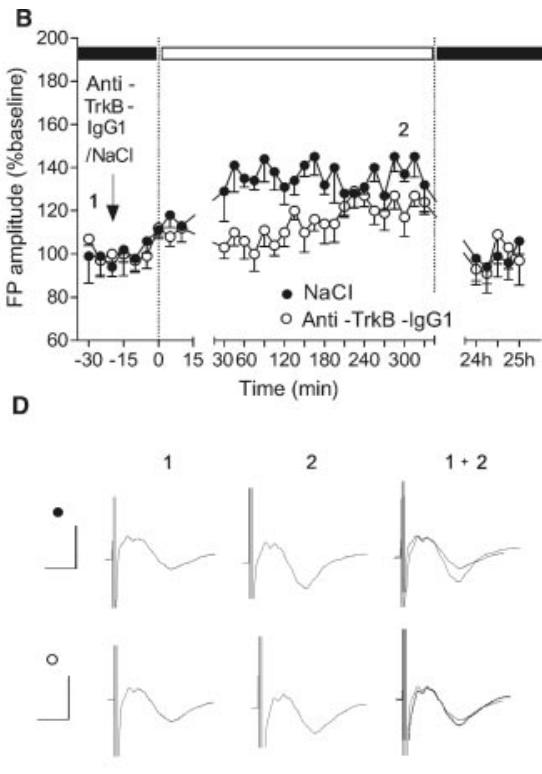

D A Anti-TrkB -lgG1 $\triangle \mathrm{NaCl}$
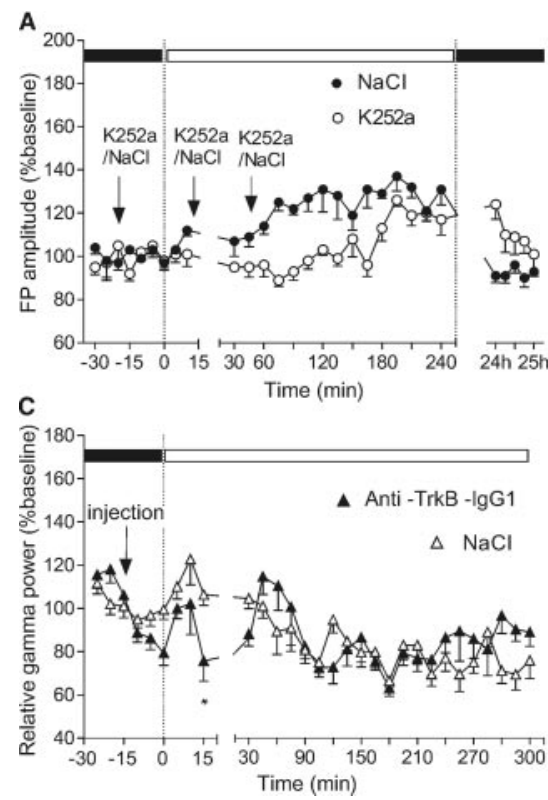
. 

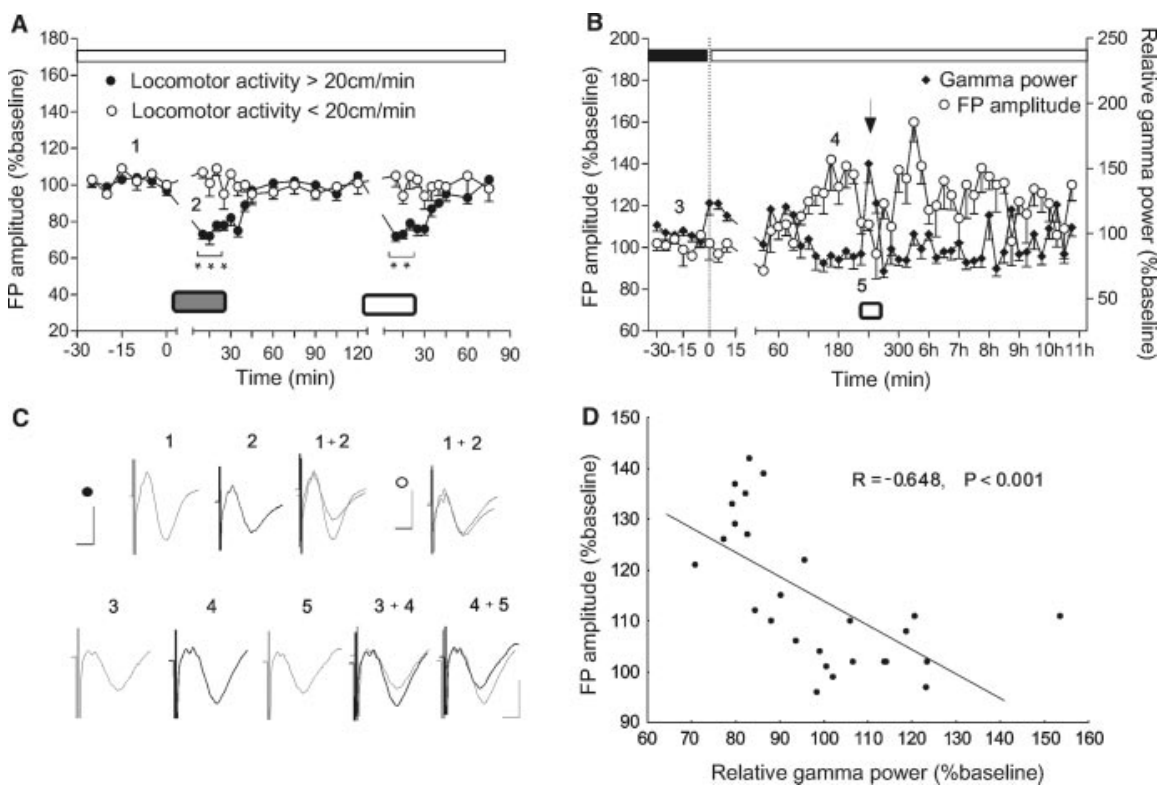

Figure 4. Visual activity parallels gamma oscillations with field potential depression. A, Arousal-induced short-term depression during high-illuminance conditions using objects of different colors (black, white, green) and shapes (cylindric or square). Animals were introduced to a toy (signified by a gray or white rectangle), starting at time 0 and for $15 \mathrm{~min}$. Depending on the arousal state, as reflected by locomotor activity, the animals responded with FP depression (locomotor activity $>20 \mathrm{~cm} / \mathrm{min}$, black dots) or with no change of the FP (locomotor activity $<20 \mathrm{~cm} / \mathrm{min}$, white dots). $\boldsymbol{B}$, The elevated phase can be interrupted by exploration-induced arousal (the arrow), coincident with robust augmentation of high-frequency oscillations. Gamma activity was enhanced during active visual exploration (white rectangle) in correlation with depressed FP amplitude. White dots represent the FP amplitude; black diamonds represent the relative values of gamma spectral power. $\boldsymbol{C}$, Analogs represent FPs evoked at the points marked in the figures. Calibration: $5 \mathrm{~ms}, 1 \mathrm{mV}$. The top left trace represents the FP changes in the aroused group (black dots) before (1) versus during the object presentation (2); the top right trace, FPs in nonaroused group (white dots). D, FP changes negatively correlate with gamma power in the visual cortex.

latory activity and the direction of change in synaptic strength elicited in the visual cortex.

Our findings support that the visual cortex undergoes naturalistic regulation of synaptic plasticity thresholds. This finding is an extension of work by others that demonstrated that, for example, dark exposure for prolonged periods leads to a shift in the thresholds for induction of LTP or long-term depression (LTD) (Kirkwood and Bear, 1994; Kirkwood et al., 1995, 1996). These findings are also in accordance with the sliding threshold model of synaptic plasticity in the visual cortex (Bienenstock et al. 1982; Bear et al. 1987). But what is truly a new insight is that our findings suggest that the visual cortex is subject to continuous metaplastic regulation that is determined by the phase of the light-dark cycle, the circadian phase triggered by this cycle, and active visual processing occurring at a given moment in time. These factors appear to be critical in engineering the sliding threshold that favors LTP or LTD induction, depending on the behavioral state of the animal.

BDNF and TrkB mRNA undergo diurnal regulation (Bova et al., 1998). Furthermore, BDNF protein is present in higher concentrations in the middle of the light phase, compared with the middle of the dark phase of the circadian cycle (Pollock et al., 2001). Our study supports that BDNF plays an important role in enabling the diurnal fluctuations of basal synaptic transmission. Inhibiting TrkB delayed the onset of field potential augmentation that typically occurred after the transition from dark to light within the $12 \mathrm{~h}$ light/dark cycle. In young animals, application of BDNF shifts the threshold for LTP induction to the left, enhances weak LTP, and cannot improve maximal LTP elicited by strong tetanization (Akaneya et al., 1997; Huber et al., 1998). A correla- tion between BDNF levels and the expression of LTP and LTD have been demonstrated, whereby increases in BDNF levels are associated with LTP induction and decreases are linked to LTD (Aicardi et al., 2004). Together, these findings suggest that BDNF may favor synaptic potentiation and minimize synaptic depression, and that changes in BDNF levels may be a critical factor in the naturalistic fluctuations of synaptic strength that we observed through the light-dark cycle.

In agreement with field potential studies in rat neocortex (Kandel and Buzsaki, 1997), our observations suggest that the visual cortex is not simply a passive receiver of sensory inputs, but can dynamically amplify oscillatory activity arising in the thalamus. Moreover, we demonstrate that visual experience can trigger plasticity processes, which, although acquired during wakefulness, may be consolidated during sleep (Steriade, 2000; Timofeev et al., 2000; Steriade and Timofeev, 2003). In our experiments, we detected an increase in $30-100 \mathrm{~Hz}$ EEG frequency in the visual cortex during visual exploration by the animals. Fast rhythms within the gammafrequency band (generally $30-80 \mathrm{~Hz}$ ), which are present in background electrical activity during the brain-activated states of waking and REM sleep, are thought to enhance temporal coherence of responses and firing probability of cortical neurons (Steriade, 2000). During theta behavioral states, a prominent enhancement of gamma oscillations (40-100 Hz) also occurs. High-frequency oscillations in cortical structures are believed to provide temporal windows that bind coherently cooperating neuronal assemblies to enable the representation, processing, storage, and retrieval of information (Buzsaki et al., 1994). Gamma oscillations have received special attention because of their alleged role in sensory binding (Singer and Gray, 1995; Gray and Singer, 1989), as well as memory and attentional selection (Fries et al., 2001). For hippocampal circuits, experimentally induced gamma oscillations produce a prolonged enhancement of recurrent excitatory connections between CA1 pyramidal neurons (Whittington et al., 1997). In the present study, gamma activity during visual exploration under high-illuminance conditions was associated with a significant increase of subsequent field potential augmentation, compared with low-illuminance conditions, in which gamma activity was not enhanced. A model consistent with the results of our study describes how sensory deprivation, for long periods, shifts the modification threshold (for induction of synaptic plasticity by postsynaptic activity) leftward, favoring induction LTP in response to synaptic activation of layer $2 / 3$ neurons (Philpot et al., 2001). Our data suggest that these effects also occur acutely and play a decisive role in the expression of subsequent plasticity.

Long-term potentiation of field potentials evoked in the primary visual cortex of adult anesthetized rats was reliably induced after theta burst stimulation of the dLGN, and was found to increase the amplitude of visually evoked potentials across a range of spatial frequencies (Heynen and Bear, 2001). In vivo, visual cortical neurons are in a steady state of either synaptic 
depression or facilitation, which serves as a background to all additional synaptic inputs (Tsanov and Manahan-Vaughan, 2007). In addition to synaptic potentiation, synaptic depression and intracortical inhibition have been proposed as mechanisms that influence the responsiveness of the primary visual cortex (Carandini et al., 2002). In the visual cortex, short-term synaptic plasticity, and in particular synaptic depression, is an important component of the nonlinear temporal dynamics that lead to enhancement of responses (Chance et al., 1998). In agreement with these findings, we detected depression of the field response in the visual cortex of freely moving rats during exploratory behavior. Short-term depression on a population level allows activation, elicited by stimulation of the brainstem reticular formation, to result in an enhanced sensitivity of single visual cortex neurons to subtle changes in the firing pattern of its afferents (Abbott et al., 1997). An important issue was the establishment of a temporal parallel between the evoked depression and the increase of power in the gamma band. Gamma oscillations contribute to maintenance of neuronal synchrony in mediating perceptual binding (Nase et al., 2003) and in enhancing the transmission of sensory information for higher cognitive processing (Samonds and Bonds 2005). We demonstrated a significant correlation between gamma oscillations and field potential depression in arousalinduced visual responses. Our findings show for the first time that network oscillatory activity and depression of field potentials may act synergistically in rodent visual cortex.

This study reports that visual experience in freely behaving animals under physiological conditions increases field responses in the visual cortex. Superimposed on an intrinsic, circadian oscillation of basal synaptic transmission driven by the light-dark cycle, active visual exploration shapes the final amplitude of the cortical field potential. The present results are consistent with the hypothesis that the adult visual cortex acutely undergoes longterm synaptic modification in response to sensory stimuli. Our data suggest that this phenomenon might be mediated through gamma rhythms in association with short-term field depression, an arousal-related response that may alter the efficacy of the visual perception by increasing the signal-to-noise ratio.

\section{References}

Abbott LF, Varela JA, Sen K, Nelson SB (1997) Synaptic depression and cortical gain control. Science 275:220-224.

Aicardi G, Argilli E, Cappello S, Santi S, Riccio M, Thoenen H, Canossa M (2004) Induction of long-term potentiation and depression is reflected by corresponding changes in secretion of endogenous brain-derived neurotrophic factor. Proc Natl Acad Sci USA 101:15788-15792.

Akaneya Y, Tsumoto T, Kinoshita S, Hatanaka H (1997) Brain-derived neurotrophic factor enhances long-term potentiation in rat visual cortex. J Neurosci 17:6707-6716.

Bear MF (1996) A synaptic basis for memory storyge in the cerebral cortex. Proc Natl Acad Sci USA 93:13453-13459.

Bear MF, Cooper LN, Ebner FF (1987) A physiological basis for a theory of synaptic modification. Science 237:42-48.

Bienenstock EL, Cooper LN, Munro PW (1982) Theory for the development of neuron selectivity: orientation specificity and binocular interaction in visual cortex. J Neurosci 2:32-48.

Bova R, Micheli MR, Qualadrucci P, Zucconi GG (1998) BDNF and trkB mRNAs oscillate in rat brain during the light-dark cycle. Mol Brain Res 57:321-324.

Buzsáki G, Llinás R, Berthoz A, Christen Y (eds) (1994) Temporal coding in the brain. Berlin: Springer.

Carandini M, Heeger DJ, Senn W (2002) A synaptic explanation of suppression in visual cortex. J Neurosci 22:10053-10065.

Castro-Alamancos MA, Connors BW (1996) Cellular mechanisms of the augmenting response: short-term plasticity in a thalamocortical pathway. J Neurosci 16:7742-7756.
Chance FS, Nelson SB, Abbott LF (1998) Synaptic depression and the temporal response characteristics of V1 cells. J Neurosci 18:4785-4799.

Figurov A, Pozzo-Miller L, Olafsson P, Wang T, Lu B (1996) Regulation of synaptic responses to high-frequency stimulation and LTP by neurotrophins in the hippocampus. Nature 381:706-709.

Fries P, Neuenschwander S, Engel AK, Goebel R, Singer W (2001) Rapid feature selective neuronal synchronization through correlated latency shifting. Nat Neurosci 4:194-200.

Gray CM, Singer W (1989) Stimulus-specific neuronal oscillations in orientation columns of cat visual cortex. Proc Natl Acad Sci USA 86:1698-1702.

Heynen AJ, Bear MF (2001) Long-term potentiation of thalamocortical transmission in the adult visual cortex in vivo. J Neurosci 21:9801-9813.

Huber KM, Sawtell NB, Bear MF (1998) Brain-derived neurotrophic factor alters the synaptic modification threshold in visual cortex. Neuropharmacology 37:571-579.

Hughes HC (1977) Anatomical and neurobehavioral investigations concerning the thalamo cortical organization of the rat's visual system. J Comp Neurol 175:311-336.

Kandel A, Buzsaki G (1997) Cellular-synaptic generation of sleep spindles, spike-and-wave discharges, and evoked thalamocortical responses in the neocortex of rat. J Neurosci 17:6783-6797.

Kemp A, Manahan-Vaughan D (2004) Hippocampal long-term depression and long-term potentiation encode different aspects of novelty acquisition. Proc Natl Acad Sci USA 101:8192-8197.

Kemp A, Manahan-Vaughan D (2005) The 5-hydroxytryptamine4 receptor exhibits frequency-dependent properties in synaptic plasticity and behavioural metaplasticity in the hippocampal CA1 region in vivo. Cereb Cortex 15:1037-1043.

Kemp A, Manahan-Vaughan D (2007) Hippocampal LTD: master or minion in declarative memory processes? Trends Neurosci 30:111-118.

Kirkwood A, Bear MF (1994) Hebbian synapses in visual cortex. J Neurosci 14:1634-1645.

Kirkwood A, Lee HK, Bear MF (1995) Co-regulation of long-term potentiation and experience-dependent plasticity in visual cortex by age and experience. Nature 375:328-331.

Kirkwood A, Rioult MG, Bear MF (1996) Experience-dependent modification of synaptic plasticity in visual cortex. Nature 381:526-528.

Kovalchuk Y, Hanse E, Kafitz W, Konnerth A (2002) Postsynaptic induction of BDNF-mediated long-term potentiation. Science 295:1729-1734.

Lumer ED, Edelman GM, Tononi G (1997) Neural dynamics in a model of the thalamocortical system. I. Layers, loops and the emergence of fast synchronous rhythms. Cereb Cortex 7:207-227.

Martin SJ, Grimwood PD, Morris RG (2000) Synaptic plasticity and memory: an evaluation of the hypothesis. Annu Rev Neurosci 23:649-711:649-711.

Meijer JH, Rietveld WJ (1989) Neurophysiology of the suprachiasmatic circadian pacemaker in rodents. Physiol Rev 69:671-707.

Messaoudi E, Bardsen K, Srebro B, Bramham CR (1998) Acute intrahippocampal infusion of BDNF induces lasting potentiation of synaptic transmission in the rat dentate gyrus. J Neurophysiol 79:496-499.

Molnar Z, Adams R, Blakemore C (1998) Mechanisms underlying the early establishment of thalamocortical connections in the rat. J Neurosci 18:5723-5745.

Nase G, Singer W, Monyer H, Engel AK (2003) Features of neuronal synchrony in mouse visual cortex. J. Neurophysiol 90:1115-1123.

Philpot BD, Aarti K, Sekhar AK, Shouval HZ, Bear MF (2001) Visual experience and deprivation bidirectionally modify the composition and function of NMDA receptors in visual cortex. Neuron 29:157-169.

Pollock GS, Vernon E, Forbes ME, Yan Q, Ma YT, Hsieh T, Robichon R, Frost DO, Johnson JE (2001) Effects of early visual experience and diurnal rhythms on BDNF mRNA and protein levels in the visual system, hippocampus, and cerebellum. J Neurosci 2:3923-3931.

Ralph MR, Foster RG, Davis FC, Menaker M (1990) Transplanted suprachiasmatic nucleus determines circadian period. Science 247:975-978.

Rodriguez R, Kallenbach U, Singer W, Munk MH (2004) Short- and long-term effects of cholinergic modulation on gamma oscillations and response synchronization in the visual cortex. J Neurosci 24:10368-10378.

Rusak B, Zucker I (1979) Neural regulation of circadian rhythms. Physiol Rev 59:449-526. 
Samonds JM, Bonds AB (2005) Gamma oscillation maintains stimulus structure-dependent synchronization in cat visual cortex. J Neurophysiol 93:223-236.

Singer W, Gray CM (1995) Visual feature integration and the temporal correlation hypothesis. A Rev Neurosci 18:555-586.

Steriade M (2001) Impact of network activities on neuronal properties in corticothalamic systems. J Neurophysiol 86:1-39.

Steriade M, Timofeev I (2003) Neuronal plasticity in thalamocortical networks during sleep and waking oscillations. Neuron 37:563-576.

Steriade M, Timofeev I, Grenier F, Dürmüller N (1998) Role of thalamic and cortical neurons in augmenting responses: dual intracellular recordings in vivo. J Neurosci 18:6425-6443.

Steriade M, Timofeev I, Grenier F (2000) Input resistance of cortical neurons during natural states of vigilance in behaving cats. Soc Neurosci Abstr 26:757.10.

Timofeev I, Steriade M (1998) Cellular mechanisms underlying intrathalamic augmenting responses of reticular and relay neurons. J Neurophysiol 79:2716-2729.
Timofeev I, Grenier F, Steriade M (2000) Impact of intrinsic properties and synaptic factors on the activity of neocortical networks in vivo. J Physiol (Paris) 94:343-355.

Tsanov M, Manahan-Vaughan D (2007) The adult visual cortex expresses dynamic synaptic plasticity that is driven by the light/dark cycle. J Neurosci 27:8414-8421.

Varela JA, Sen K, Gibson J, Fost J, Abbott LF, Nelson SB (1997) A quantitative description of short-term plasticity at excitatory synapses in layer $2 / 3$ of rat primary visual cortex. J Neurosci 17:7926-7940.

Whittington MA, Traub RD, Faulkner HJ, Stanford IM, Jefferys JGR (1997) Recurrent excitatory postsynaptic potential induced by synchronized fas cortical oscillations. Proc Natl Acad Sci USA 94:12198-12203.

Ying SW, Futter M, Rosenblum K, Webber MJ, Hunt SP, Bliss TV, Bramham CR (2002) Brain-derived neurotrophic factor induces long-term potentiation in intact adult hippocampus: requirement for ERK activation coupled to CREB and upregulation of Arc synthesis. J Neurosci 22:1532-1540.

Zilles K (1985) The cortex of the rat. Berlin: Springer. 